\title{
Long non-coding RNA LSINCT5 promotes ovarian cancer cell proliferation, migration and invasion by disrupting the CXCL12/CXCR4 signalling axis
}

\author{
XINGTAO LONG ${ }^{1,2}$, LI LI $^{1 *}$, QI ZHOU ${ }^{2 *}$, HAIXIA WANG $^{2}$, DONGLING ZOU ${ }^{2}$, \\ DONG WANG $^{2}$, MENG LOU ${ }^{1}$ and WEIQI NIAN ${ }^{3}$ \\ ${ }^{1}$ Department of Gynecologic Oncology, Affiliated Tumor Hospital of Guangxi Medical University, \\ Nanning, Guangxi 530021; ${ }^{2}$ Department of Gynecologic Oncology; ${ }^{3}$ Key Laboratory of Oncology, Chongqing University \\ Cancer Hospital \& Chongqing Cancer Institute \& Chongqing Cancer Hospital, Chongqing 400030, P.R. China
}

Received June 26, 2017; Accepted February 28, 2018

DOI: $10.3892 / \mathrm{ol} .2018 .8241$

\begin{abstract}
Long stress-induced noncoding transcript 5 (LSINCT5) is a member of the LSINCT family, members of which are expressed during stress-induced cell formation and have also been reported to promote cancer progression. In the present study, the association between LSINCT5 expression and clinical significance was investigated and the biological function of LSINCT5 in epithelial ovarian cancer (EOC) was explored. LSINCT5 expression was examined in EOC tissues by reverse transcription-quantitative polymerase chain reaction and its association with clinicopathological factors was analysed. Cell proliferation, migration and invasion tests were performed to observe the role of LSINCT5 in human ovarian cancer cell lines in vitro. The negative control (NC) and siLSINCT5 SKOV3 cells were treated with chemokine ligand 12 (CXCL12) and their proliferation, migration and invasion activities were examined. LSINCT5 was overexpressed in EOC compared with normal ovarian tissue. LSINCT5
\end{abstract}

Correspondence to: Dr Li Li, Department of Gynecologic Oncology, Affiliated Tumor Hospital of Guangxi Medical University, 71 He Di Road, Nanning, Guangxi 530021, P.R. China E-mail: 1ili@gxmu.edu.cn

Dr Qi Zhou, Department of Gynecologic Oncology, Chongqing University Cancer Hospital \& Chongqing Cancer Institute \& Chongqing Cancer Hospital, 181 Han Yu Road, Chongqing 400030, P.R. China

E-mail: qizhou9128@163.com

"Contributed equally

Abbreviations: LSINCT5, long stress-induced noncoding transcript5; EOC, epithelial ovarian cancer; RT-qPCR, reverse transcription-quantitative polymerase chin reaction; CXCL12, chemokine ligand 12; FIGO, International Federation of Gynecologists and Obstetricians; CXCR4, chemokine receptor 4

Key words: IncRNA, ovarian cancer, LSINCT5, CXCL12/CXCR4, RNAi expression was significantly associated with the International Federation of Gynecologists and Obstetricians cancer stage and the presence of lymphatic metastases. Silencing LSINCT5 significantly reduced the expression of chemokine receptor 4 (CXCR4) and inhibited SKOV3 cell proliferation, migration and invasion, however the CXCL12 expression level had no significant change. When NC and siLSINCT5-SKOV3 cells were treated with CXCL12, the proliferation and invasion ability were significantly enhanced. The migration ability of the siLSINCT5-SKOV3 cells was also significantly enhanced. The present study indicated that LSINCT5 serves an important role in ovarian cancer metastasis by regulating the CXCL12/CXCR4 signalling axis, suggesting that this pathway may be a potential target for the treatment of patients with EOC.

\section{Introduction}

Epithelial ovarian cancer (EOC) is characterized by frequent implantation and metastases in the abdominopelvic cavity. Approximately $70 \%$ of EOC patients who have already distant metastasis are finally diagnosed with advanced ovarian cancer. Despite advances in treatments for ovarian cancer, including surgery, chemotherapy and targeted therapy, the 5-year survival rate of advanced ovarian cancer is only approximately $30 \%$. EOC has the highest mortality rate among all gynaecological malignancies $(1,2)$. Metastasis and invasion in ovarian cancer are the main causes of poor prognosis, but the mechanisms driving these processes remain unclear. Therefore, successfully treating this disease requires further understanding of the mechanisms promoting metastasis and invasion in EOC.

Long non-coding RNAs (lncRNAs) are a new class of non-coding RNAs and are emerging as novel regulators of many aspects of gene expression regulation including epigenetic regulation, transcriptional regulation, and post-transcriptional regulation (3). It has previously been demonstrated that IncRNAs are involved in the regulation of tumour metastasis and invasion (4-6). For example, HOTAIR has been shown to promote metastasis in breast and colorectal cancer, as well as in other malignant tumours $(7,8)$. The low 
expression of IncRNA-HEIH has been shown to inhibit liver cancer cell proliferation (9). In contrast, high expression of the lncRNA-maternally expressed gene 3 (MEG3) promotes gastric cancer cell apoptosis and inhibits proliferation (10). Emerging evidence indicates that dysregulated LSINCT5 regulates ovarian and breast cancer cell proliferation (11). However, the relationship between overexpression of LSINCT5 and ovarian cancer malignancy and metastasis remains unknown.

In this study, we measured the expression of LSINCT5 in ovarian cancer tissue samples and observed the correlation between LSINCT5 expression and clinical pathologic parameters. Moreover, we studied the effect of LSINCT5 on cancer cell metastasis and invasion in vitro. These results will help delineate the role of LSINCT5 in EOC metastatic progression.

\section{Materials and methods}

EOC and normal ovary tissue samples. Forty EOC tissue specimens and thirty normal ovary specimens were obtained from the Department of Gynecologic Oncology at Chongqing Cancer Hospital between January 2014 and September 2015. The tissue specimens were immediately flash-frozen in liquid nitrogen after being isolated. The forty EOC specimens were histopathologically confirmed by Cytoreductive surgery for ovarian cancer. All patients underwent lymphadenectomy and had not any preoperative therapy. According to the International Federation of Gynecologists and Obstetricians (FIGO) staging standard. Patients with other malignant tumours or borderline malignant tumours were excluded from the study. Thirty specimens of normal ovary were obtained from uterine fibroid patients undergoing hysterectomy, which were verified by pathology. Patients with ovarian cysts, polycystic ovaries or other disorders were excluded. Patient data were obtained from medical records and are summarized in Table I.

Ethical approval. The present study were approved by the Ethics Committee of Chongqing Cancer Hospital, and all patients gave informed consent.

Cell lines and transfection. Human ovarian cancer cell lines (SKOV3, OVCAR-3 and 3AO) were obtained from the Chongqing Key Laboratory of Oncology and were cultured in RPMI-1640 media (Wuhan Boster Biological Technology, Ltd., Wuhan, China) containing 10\% foetal bovine serum (FBS) (Wuhan Boster Biological Technology, Ltd.), penicillin and streptomycin at $37^{\circ} \mathrm{C}$ in a $5 \% \mathrm{CO}_{2}$ incubator. The LSINCT5-specific small interfering RNAs (siRNAs) and negative control (NC) siRNAs (GenePharma, Shanghai, China) were transfected to SKOV3 cells by following the manufacturers manual. The target sequences for the LSINCT5 siRNAs were 5'-CCAGCUACAAACCUCUGAATT-3' and 5'-UUCAGA GGUUUGUAGCUGGTT-3' (LSINCT5-siRNA-1); 5'-GAA CUGGAUUAGUGUUAAATT-3' and 5'-UUUAACACUAAU CCAGUUCTT-3' (LSINCT5-siRNA-2); 5'-CCUCCAAAC ACAUGGAUAATT-3' and 5'-UUAUCCAUGUGUUUGGAG GTT-3' (LSINCT5-siRNA-3).

Scratch assay. SKOV3 cells were first transfected with LSINCT5-siRNAs or siRNA-NC and then plated as a confluent monolayer in 6-well plates. When cells confluence reached to about $80 \%$, 'wounds' were created using a pipette tip in the confluent cells. The cells were rinsed 3 times with PBS to remove the floating cells displaced by the pipette tip, then media with or without Chemokine ligand 12 (CXCL12) (40 ng/ml) (PeproTech, Inc., Rocky Hill, NJ, USA) was added to the cells. Cells were placed in a $37^{\circ} \mathrm{C}, 5 \% \mathrm{CO}_{2}$ incubator for the duration of the assay. Wound healing were observed along the scrape line. Migration area were imaged using a 10x objective on an Olympus microscope and the area ratio was measured by ImageJ Software (National Institutes of Health, Bethesda, MD, USA) at 0 and 48 h. Each experiment was performed in triplicate.

Transwell invasion assay. SKOV3 cells were transfected with LSINCT5-siRNAs or siRNA-NC for $48 \mathrm{~h}$, and then, media with or without CXCL12 (40 ng/ml) was added to the cells. Cells were then placed in a $37^{\circ} \mathrm{C}, 5 \% \mathrm{CO}_{2}$ incubator for $48 \mathrm{~h}$. The transfected cells $\left(1.0 \times 10^{5}\right)$ were harvested and then seeded into the top chamber of inserts containing $8 \mu \mathrm{m}$ pore polycarbonate filters (Corning Incorporated, Corning, NY, USA), which was coated with Matrigel membrane (BD Biosciences, Franklin Lakes, NJ, USA). Experiments were performed in triplicate. The inserts were placed inside 24 -well plates. After $48 \mathrm{~h}$, the cells that failed to migrate through the pores were discarded and the invaded cells were stained with crystal violet (Beyotime Institute of Biotechnology, Beijing, China) and counted under a microscope (magnification, x200).

Cell proliferation. Cell proliferation was measured using the CCK8 kit (Biyuntian, Shanghai, China) according to the manufacturer's instructions. SKOV3 cells were seeded into 96-well plates at a density of $5 \times 10^{3}$ cells/well and incubated for $24 \mathrm{~h}$. At $48 \mathrm{~h}$ post transfection with LSINCT5-siRNAs or siRNA-NC, media with or without CXCL12 (40 ng/ml) was added in a $37^{\circ} \mathrm{C}, 5 \% \mathrm{CO}_{2}$ incubator for $48 \mathrm{~h}$. CCK-8 solution was then added to the cells, and the optical density was measured by a microplate reader at an absorbance of $450 \mathrm{~nm}$. Three independent experiments were performed.

$R N A$ isolation and $q P C R$. Total cellular RNA was extracted from EOC tissues and human ovarian cancer cell lines using Trizol reagent (Takara Bio, Inc., Otsu, Japan) and purified using an RNA Purification kit (Takara Bio, Inc.). Reverse transcription was performed using PrimeScript RT master Mix (Takara Bio, Inc.). qPCR was performed using a SYBR-Green MIX kit (Bio-Rad Laboratories, Inc., Hercules, CA, USA). The primer sequences used for human LSINCT5 are as follows: Forward, 5'-CCAGCUACAAACCUCUGAATT-3', and reverse, 5'-UUCAGAGGUUUGUAGCUGGTT-3'. GAPDH (sequences: Forward, 5'-AGGGCTGCTTTTAACTCTGGT-3', and reverse, 5'-CCCCACTTGATTTTGGAGGGA-3') was used as an internal standard, the relative expression of each gene was normalized to GAPDH. PCR cycling conditions were as follows: $95^{\circ} \mathrm{C}$ for $5 \mathrm{~min}$, followed by 40 cycles of $95^{\circ} \mathrm{C}$ for $30 \mathrm{sec}, 60^{\circ} \mathrm{C}$ for $20 \mathrm{sec}$ and $72^{\circ} \mathrm{C}$ for $20 \mathrm{sec}$. Relative quantification of mRNA was performed using comparative threshold cycle (CT) method. This value was used to plot the gene expression employing the formula $2^{-\Delta \Delta \mathrm{Cq}}(12)$.

Western blot analysis. SKOV3 cells were transfected with LSINCT5-siRNAs or LSINCT5-NC, and total protein 
Table I. The correlation between LSINCT5 expression and clinical pathologic parameters in 40 patients with EOC.

\begin{tabular}{lccc}
\hline & \multicolumn{3}{c}{ LSINCT5 expression } \\
\cline { 2 - 4 } Variables & $\begin{array}{c}\text { Low no. } \mathrm{n}=20 \\
\mathrm{n}(\%)\end{array}$ & $\begin{array}{c}\text { High no. } \mathrm{n}=20 \\
\mathrm{n}(\%)\end{array}$ & P-value \\
\hline Age (years) & & & 0.749 \\
$<50$ & $8(47.1)$ & $9(52.9)$ & \\
$\geq 50$ & $12(52.2)$ & $11(47.8)$ & \\
FIGO stage & & & 0.004 \\
I-II & $13(76.5)$ & $4(23.5)$ & \\
III-IV & $7(30.4)$ & $16(69.6)$ & \\
Grade & & & 0.736 \\
G1-G2 & $7(53.8)$ & $6(46.2)$ & \\
G3 & $13(48.1)$ & $14(51.9)$ & \\
Residual tumor & & & 0.490 \\
diameter (cm) & & & \\
$<1$ & $15(53.6)$ & $13(46.4)$ & \\
$\geq 1$ & $5(41.7)$ & $7(58.3)$ & \\
Lymph node & & & \\
metastasis & & & \\
Absent & & & \\
Present & $14(87.5)$ & $(12.5)$ & \\
Histological & & & \\
subtype & & & \\
Serous & & & \\
Other & & & \\
\hline
\end{tabular}

${ }^{\text {a}}$ Fisher's exact test. LSINCT5, long stress-induced noncoding transcript5; FIGO, International Federation of Gynecology and Obstetrics.

was isolated from the cells $48 \mathrm{~h}$ post transfection with cell lysis buffer (Beyotime Institute of Biotechnology). Protein concentrations were determined by BCA (Beyotime Institute of Biotechnology). Protein samples $(40 \mu \mathrm{g})$ were run on an SDS-PAGE gel and then transferred to a PVDF membrane (EMD Millipore, Billerica, MA, USA). The membranes were blocked with $5 \%$ skimmed milk for $2 \mathrm{~h}$ and then incubated with anti-chemokine C-X-C Motif receptor 4 (CXCR4) antibody (1:600; ab124824, Abcam, Cambridge, MA, USA) and Anti-SDF1 antibody (1:1,000; ab9797, Abcam) at $4^{\circ} \mathrm{C}$ overnight. GAPDH (1:1,000, D110016; Sangon Biotech Co., Ltd., Shanghai, China) was used as the loading control. All the antibodies were diluted by specific dilution designed for western blot antibody (Boster Biological Technology, Pleasanton, CA, USA). The membrane was then incubated with secondary antibody for $2 \mathrm{~h}$ and visualized with Enhanced chemiluminescence (ECL) (Beyotime Institute of Biotechnology).

Statistical analyses. SPSS19 statistic software was used for all data analyses. Student's t-test or analysis of variance followed by Turkey's post hoc test was performed to analyse continuous data, a Chi-Square test was performed to analyse categorical data between groups. Fisher's exact test was performed when the expected frequency was less than five. $\mathrm{P}<0.05$ was considered to indicate a statistically significant difference.

\section{Results}

LSINCT5 sequence comparison. The sequence of LSINCT5 was compared on three databases (lncrnadb, noncode and ensembl). The LSINCT5 sequence is completely consistent with the published sequence. We used Genome Bioinformatics tools (UCSC Table Browser) to check the conservation. Input lncRNA chr5:2,712,705-2,715,351 position information. The results show conservation information on multiple vertebrates. In the present study, EOC tissues and SKOV3 cells are derived from humans, so the sequence of LSINCT5 are highly conservative between species and cell type.

LSINCT5 is overexpressed in EOC tissues. The expression levels of LSINCT5 in 40 EOC and 30 normal ovarian tissue samples were evaluated using qRT-PCR. LSINCT5 expression was largely elevated in EOC tissues compared to control normal tissues $(\mathrm{P}<0.01$; Fig. $1 \mathrm{~A})$.

The relationship between LSINCT5 expression and clinical pathological factors. The median value of LSINCT5 expression in the EOC tissues was determined. EOC samples with LSINCT5 expression less than or equal to the median were considered low expression samples ( $n=20$ EOC samples), while EOC samples with LSINCT5 expression levels above the median were considered high expression samples. High LSINCT5 expression was associated with the presence of lymphatic metastases and advanced FIGO stage, but was not associated with patient age, histological subtype, histological grade or residual tumour diameter (Pearson's Chi-squared test or Fisher's exact tes; Table I).

LSINCT5 expression levels in human ovarian cancer cell lines. Because we found that LSINCT5 expression was associated with the presence of lymphatic metastases and FIGO stage in EOC, we hypothesized that LSINCT5 is involved in EOC invasion and metastasis. Therefore, we determined the LSINCT5 expression levels in EOC cell lines with different invasive abilities (SKOV3, OVCAR-3 and 3AO). Although all three cell lines expressed LSINCT5, SKOV3, a highly invasive EOC cell line, had the highest LSINCT5 expression (Fig. 1B). Therefore, we chose to perform in vitro experiments with SKOV3 cells. We tested three different si-LSINCT5 sequences to use for our experiments and found LSINCT5-siRNA-1 was the best choice (Fig. 1C) and was therefore used in all the following experiments.

LSINCT5 silencing reduces ovarian cancer cell proliferation, migration and invasion. Upon LSINCT5 silencing, we found a significant decrease in cancer cell proliferation (Fig. 2A). LSINCT5-silencing also led to decreased migration (Fig. 2B) and invasion (Fig. 2C) of SKOV3 cells during a wound healing assay and a transwell invasion assay, respectively.

LSINCT5 knockdown suppresses the CXCL12/CXCR4 signalling axis. It has previously been reported that CXCR4 

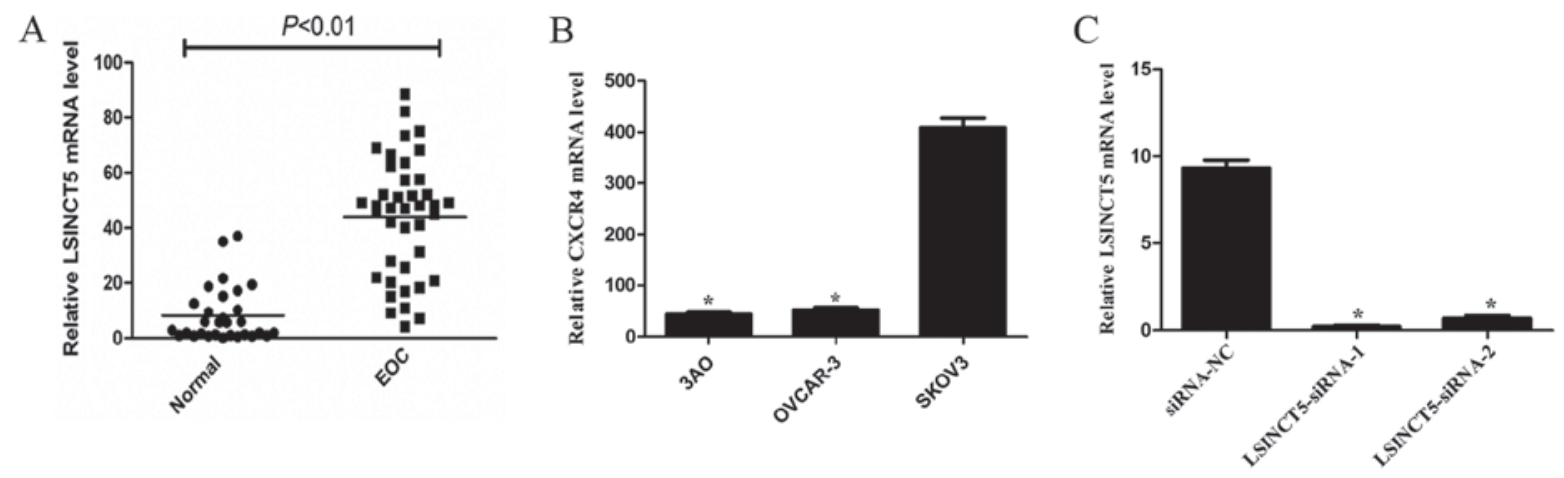

Figure 1. LSINCT5 expression in EOC tissues and cell lines. (A) Relative LSINCT5 expression levels in EOC tissues and normal ovarian tissues (P $<0.01$, Student's t-test). (B) The highest expression level was detected in SKOV3 cells. ${ }^{*} \mathrm{P}<0.05$ vs. the SKOV3 (C) LSINCT5 interference efficiency in SKOV3 cells. ${ }^{*} \mathrm{P}<0.05$ vs. the siRNA-NC.

A

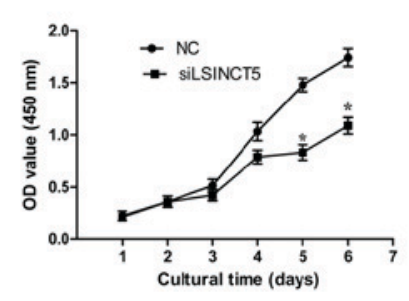

B

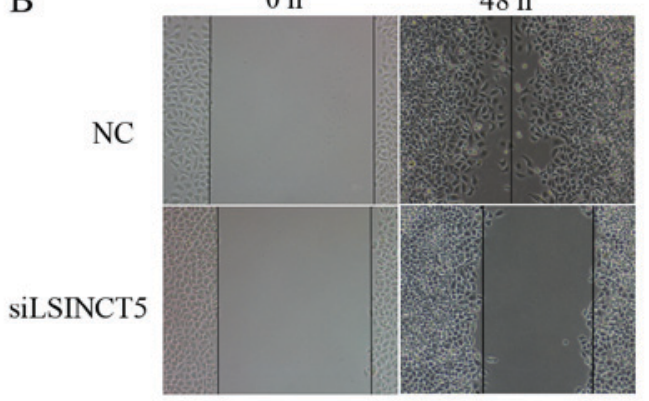

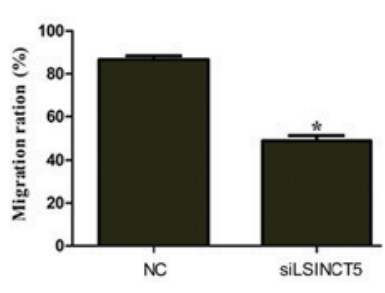
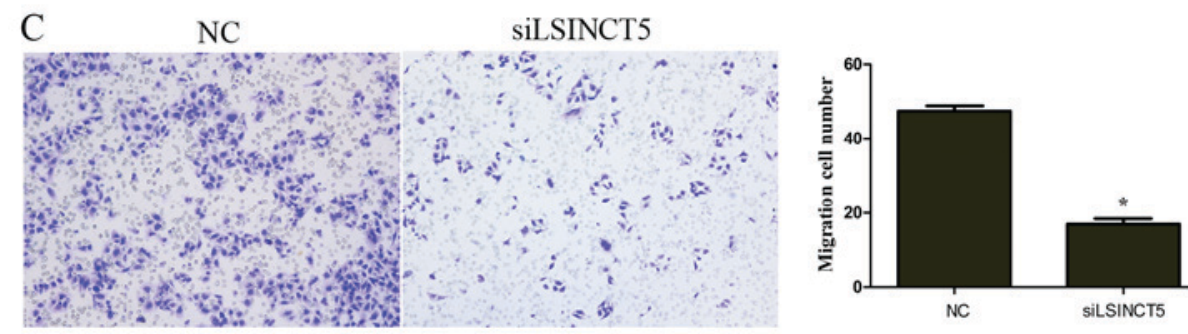

Figure 2. The effect of suppression of LSINCT5 expression on cell growth, migration, and invasion. (A) CCK-8 assay. Suppression of LSINCT5 expression inhibits SKOV3 cell growth from day 2. (B) Scratch assay. Suppression of LSINCT5 expression inhibits SKOV3 cell migration. (C) Transwell invasion assay. Suppression of LSINCT5 expression inhibits SKOV3 cell invasion. Magnification, $\mathrm{x} 200$. "P<0.05 vs. the NC.

expression is decreased by more than two-fold upon LSINCT5 knockdown, and CXCR4 has been shown to play an important role in ovarian cancer metastasis by binding to its receptor CXCL12. This prompted us to investigate if LSINCT5 promotes ovarian cancer cell metastasis by regulating the CXCL12/CXCR4 signalling axis. LSINCT5 silencing suppressed CXCR4 mRNA ("P<0.05, Fig. 3A) and protein expression $(\mathrm{P}<0.05$, Fig. $3 \mathrm{~B})$, but the CXCL12 mRNA (P>0.05, Fig. 3C) and protein expression level $(\mathrm{P}>0.05$, Fig. 3D) had no significant change. When the NC and siLSINCT5 SKOV3 cells were exposed to CXCL12 $(40 \mathrm{ng} / \mathrm{ml})$, the $\mathrm{NC}$ cell proliferation ability was stronger than that of the control group (NC cells without CXCL12 exposure) by CCK- 8 assay ( ${ }^{*} \mathrm{P}<0.01$, Fig. $\left.4 \mathrm{~A}\right)$, the si-LSINCT5 SKOV3 cells proliferation ability was also stronger than that of the control group (si-LSINCT5 SKOV3 cells without CXCL12 exposure) ( ${ }^{* *} \mathrm{P}<0.05$, Fig. 4A). CXCL12 treatment significantly increased migration in a scratch assay for si-LSINCT5 SKOV3 cells than that of the control group
(si-LSINCT5 SKOV3 cells without CXCL12 exposure) $($ ( $\mathrm{P}<0.05$, Fig. 4B), however, the migration of $\mathrm{NC}$ cell did not change significantly than that of the control group (NC cells without CXCL12 exposure) (P>0.05, Fig. 4B). CXCL12 treatment significantly increased invasion in a transwell invasion assay for both $\mathrm{NC}\left({ }^{*} \mathrm{P}<0.05\right.$, Fig. 4C) and si-LSINCT5 SKOV3 cells $\left({ }^{* *} \mathrm{P}<0.05\right.$, Fig. $\left.4 \mathrm{C}\right)$.

\section{Discussion}

LSINCT5 is a newly discovered member of the stress and cancer-associated lncRNA family, the members of which are expressed by normal human bronchial epithelial (NHBE) cells treated with the tobacco carcino-gennitrosamine 4-(methyl-nitrosamino)-1- (3-pyridyl)-1-buta-none (NNK) (11). Previous studies have reported LSINCT5 gene sequences, furthermore, high LSINCT5 expression has been associated with tumour cell metastasis in multiple cancers, including breast cancer and gastric cancer $(11,13)$. Although the study 


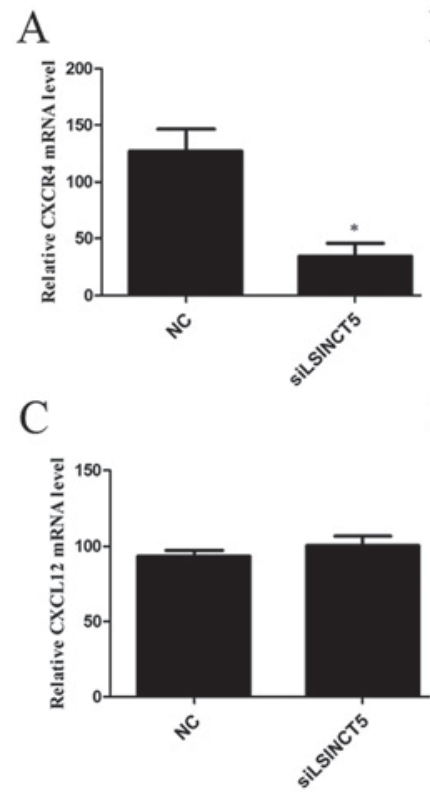

B

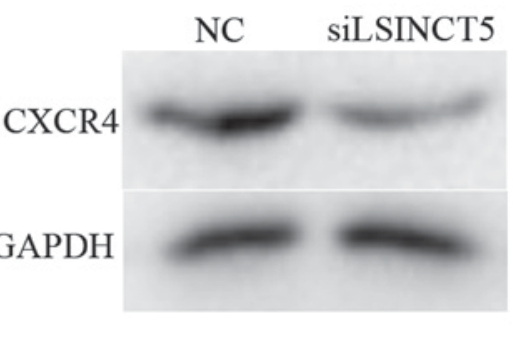

D

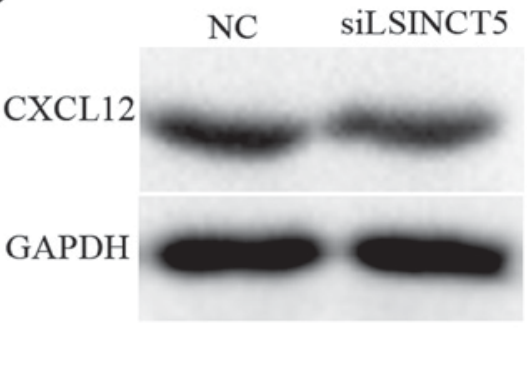

Figure 3. The effect of silencing LSINCT5 on the expression of CXCR4 and CXCL12. (A) the expression level of CXCR4 RNA. (B) The expression level of CXCR4 protein. (C) The expression level of CXCL12 RNA. (D) The expression level of CXCL12 protein. ${ }^{*} \mathrm{P}<0.05$ vs. NC.
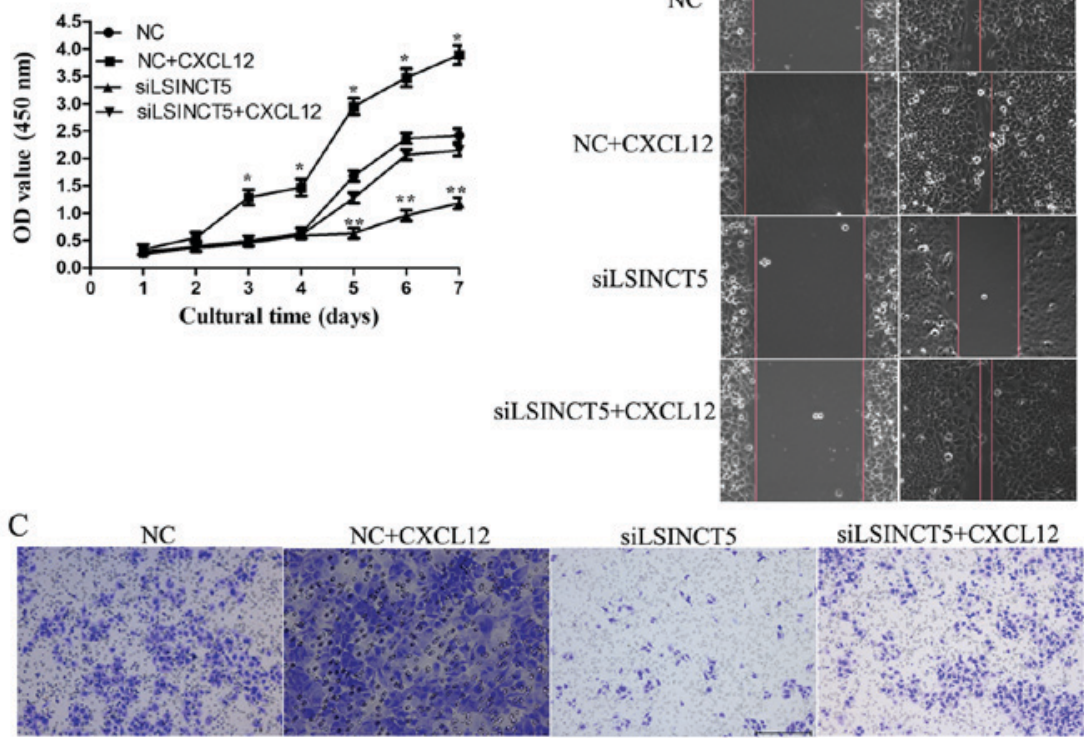

B
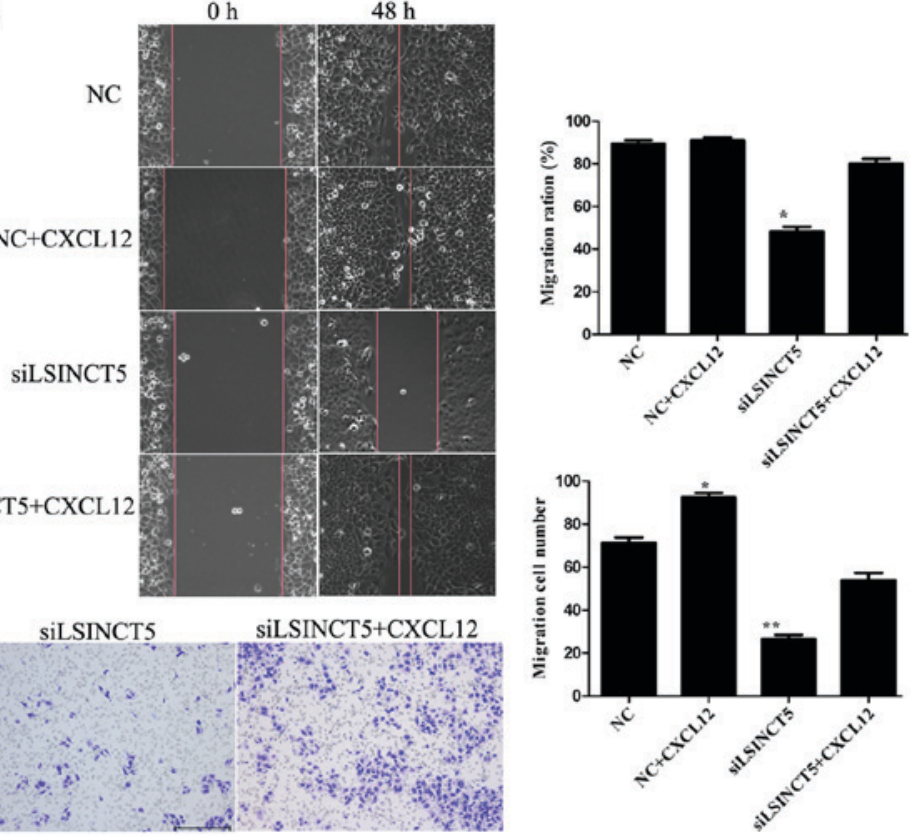

Figure 4. The effect of the si-LSINCT5 and si-NC SKOV3 cells were exposed to CXCL12 on cell growth, migration, and invasion. (A) CCK-8 assay. Exposing to CXCL12 resulted in significantly enhanced cell growth from day 3 and 5 . ${ }^{*} \mathrm{P}<0.01 \mathrm{vs.} \mathrm{NC}$; ${ }^{* *} \mathrm{P}<0.05$ vs. siLSINCT5+CXCL12. (B) Scratch assay. Exposing to CXCL12 resulted in no significantly enhanced cell migration for NC cells, but resulted in significantly enhanced cell migration for si-LSINCT5 SKOV3 cells. "P<0.05 vs. the siLSINCT5+CXCL12. (C) Transwell invasion assay. Exposing to CXCL12 resulted in significantly enhanced cell invasion for both NC and si-LSINCT5 SKOV3 cells. Magnification, $\mathrm{x} 200 .{ }^{*} \mathrm{P}<0.05$ vs. the NC. ${ }^{* *} \mathrm{P}<0.05$ vs. the siLSINCT5+CXCL12.

has suggested that LSINCT5 expression is markedly high in EOC tissue, but the relationships among LSINCT5 expression, LSINCT5 biological function and EOC clinicopathological characteristics remain unclear.

Here, we observed that LSINCT5 is overexpressed in EOC tissues and a human EOC line with high metastatic potential (SKOV3). LSINCT5 overexpression was associated with advanced FIGO stage and the occurrence of lymphatic metastasis. These results suggest that aberrantly high expression of
LSINCT5 is correlated with EOC metastasis. In this study, EOC tissues and SKOV3 cells are derived from humans, so the sequence of LSINCT5 are highly conservative between species and cell type.

Recent evidence has suggested that lncRNAs are vital regulators of many cellular stressors and that stress-responsive lncRNAs may be linked to cancer metastasis and development $(14,15)$. The upregulation of lincRNA-ROR has been shown to be important for human breast cancer invasion (16). 
lincRNA-ROR also played a significant role in upregulating HIF-1 $\alpha$ protein under stressful conditions in hepatocellular cancer (HCC) cells $(17,18)$ lncRNA C17orf91 has been implicated in the regulation of ovarian cancer cell migration and invasion (19). Pint lincRNA is lowly expressed in primary colonic cancer, and its high expression has been shown to reduce the proliferation of colon cancer cells, indicating that Pint is a potential tumour suppressor (20). In the present study, because LSINCT5 expression was closely associated with advanced EOC stage and the occurrence of lymphatic metastasis, we assessed whether LSINCT5 promotes the invasion and migration of ovarian cancer cells. We found that silencing LSINCT5 expression in vitro suppressed SKOV3 cellular proliferation and invasion, suggesting that LSINCT5 may play an important role in EOC metastasis.

The mechanism by which lncRNAs promote tumour cell metastasis and invasion remains unclear. Recent studies have postulated that IncRNAs contribute to cancer progression by regulating protein coding gene expression at the epigenetic, transcriptional, posttranscriptional, and other levels (21-23). For example, HOTAIR silencing in an HCC cell line reduced the expression of vascular endothelial growth factor (VEGF) and matrix metalloproteinase-9 (MMP-9) and consequently inhibited cell motility and metastatic ability (24). HOTAIR has also been shown to inhibit RNA binding motif 38 (RBM38) protein expression and initiate HCC cell invasion and migration (25). The MALAT1 target gene PRKA kinase anchor protein 9 (AKAP-9) has been shown to be overexpressed in both colon and colorectal cancer cells with metastatic potential (26). Due to its highly invasive and metastatic nature, EOC is considered the most lethal of all gynaecological cancers, but the mechanisms driving these processes are still unclear (27). Our results prompted us to investigate whether the roles of LSINCT5 in cellular invasion and metastasis are due to its regulation of the expression of metastasis-promoting proteins. We found that knockdown of LSINCT5 suppressed the expression of the CXCR4 in SKOV3 cells. CXCR4 is a stromal cell-derived factor-1 receptor that is significantly overexpressed in many cancer cell types, such as ovarian cancer, breast cancer and leukaemia $(28,29)$. Recent studies have demonstrated that CXCR4 and its ligand chemokine ligand (CXCL)-12, also known as SDF-1, contribute to cancer cell proliferation, migration and invasion in multiple solid tumours, including ovarian cancer $(30,31)$. The present study showed that LSINCT5 knockdown significantly downregulated CXCR4 gene and protein expression in SKOV3 cells. CXCR4 expression was decreased by silencing of LSINCT5 for $48 \mathrm{~h}$, but cell proliferation ability had no significant difference until the fifth day. We speculate there may be no synchronization between gene or protein changes and biological behavior. LSINCT5 silencing inhibited the expression of CXCR4, followed by a change in biological behavior. Other studies also showed cell invasion and metastasis ability varied within $48 \mathrm{~h}$, but proliferation ability significant differences only fifth days later (19,32).

Furthermore, CXCL12 signaling in conjunction with CXCR4 signaling has been implicated in the progression of ovarian cancer. The present study shows that CXCL12 expression is not suppressed by knockdown of LSINCT5. But when the NC and si-LSINCT5 SKOV3 cells were treated with exogenous CXCL12, the cell proliferation and invasion ability were increased compared to control group. These results suggest that LSINCT5 promotes SKOV3 cell invasion and metastasis by activating the CXCR4/CXCL12 axis.

In summary, the LSINCT5/CXCR4/SDF-1 axis plays a key role in EOC cellular proliferation, migration and invasion. These results suggest that LSINCT5-based therapeutic strategies may show promise for EOC patients.

\section{Acknowledgements}

The present study was supported by the Fundamental and Frontier Sciences research Project of Science and Technology of Chongqing (grant no. cstc2015jcyjB0601).

\section{References}

1. Siegel RL, Miller KD and Jemal A: Cancer statistics, 2017. CA Cancer J Clin 67: 7-30, 2017.

2. Ng JS, Low JJ and Ilancheran A: Epithelial ovarian cancer. Best Pract Res Clin Obstet Gynaecol 26: 337-345, 2012.

3. Zhang R, Xia LQ, Lu WW, Zhang J and Zhu JS: LncRNAs and cancer. Oncol Lett 12: 1233-1239, 2016.

4. Matouk IJ, DeGroot N, Mezan S, Ayesh S, Abu-lail R, Hochberg A and Galun E: The H19 non-coding RNA is essential for human tumor growth. PLoS One 2: e845, 2007.

5. Zhou Y, Zhang X and Klibanski A: MEG3 noncoding RNA: A tumor suppressor. J Mol Endocrinol 48: R45-R53, 2012.

6. Huarte M, Guttman M, Feldser D, Garber M, Koziol MJ, Kenzelmann-Broz D, Khalil AM, Zuk O, Amit I, Rabani M, et al: A large intergenic noncoding RNA induced by p53 mediates global gene repression in the p53 response. Cell 142: 409-419, 2010.

7. Gupta RA, Shah N, Wang KC, Kim J, Horlings HM, Wong DJ, Tsai MC, Hung T, Argani P, Rinn JL, et al: Long non-coding RNA HOTAIR reprograms chromatin state to promote cancer metastasis. Nature 464: 1071-1076, 2010.

8. Yao Y, Li J and Wang L: Large intervening non-coding RNA HOTAIR is an indicator of poor prognosis and a therapeutic target in human cancers. Int J Mol Sci 15: 18985-18999, 2014

9. Yang F, Zhang L, Huo XS, Yuan JH, Xu D, Yuan SX, Zhu N, Zhou WP, Yang GS, Wang YZ, et al: Long noncoding RNA high expression in hepatocellular carcinoma facilitates tumor growth through enhancer of zeste homolog 2 in humans. Hepatology 54: 1679-1689, 2011.

10. Sun M, Xia R, Jin F, Xu T, Liu Z, De W and Liu X: Downregulated long noncoding RNA MEG3 is associated with poor prognosis and promotes cell proliferation in gastric cancer. Tumour Biol 35: 1065-1073, 2014

11. Silva JM, Boczek NJ, Berres MW, Ma X and Smith DI: LSINCT5 is over expressed in breast and ovarian cancer and affects cellular proliferation. RNA Biol 8: 496-505, 2011.

12. Fendri A, Kontos CK, Khabir A, Mokdad-Gargouri R and Scorilas A: BCL2L12 is a novel biomarker for the prediction of short-term relapse in nasopharyngeal carcinoma. Mol Med 17: 163-171, 2011.

13. Xu MD, Qi P, Weng WW, Shen XH, Ni SJ, Dong L, Huang D, Tan C, Sheng WQ, Zhou XY and Du X: Long non-coding RNA LSINCT5 predicts negative prognosis and exhibits oncogenic activity in gastric cancer. Medicine (Baltimore) 93: e303, 2014.

14. Sonkoly E, Bata-Csorgo Z, Pivarcsi A, Polyanka H, Kenderessy-Szabo A, Molnar G, Szentpali K, Bari L, Megyeri K, Mandi Y, et al: Identification and characterization of a novel, psoriasis susceptibility-related noncoding RNA gene, PRINS. J Biol Chem 280: 24159-24167, 2005.

15. Szegedi K, Sonkoly E, Nagy N, Németh IB, Bata-Csörgo Z, Kemény L, Dobozy A and Széll M: The anti-apoptotic protein G1P3 is overexpressed in psoriasis and regulated by the non-coding RNA, PRINS. Exp Dermatol 19: 269-278, 2010.

16. Chen YM, Liu Y, Wei HY, Lv KZ and Fu P: Linc-ROR induces epithelial-mesenchymal transition and contributes to drug resistance and invasion of breast cancer cells. Tumour Biol 37: 10861-10870, 2016.

17. Takahashi K, Yan IK, Haga $\mathrm{H}$ and Patel T: Modulation of hypoxia-signaling pathways by extracellular linc-RoR. J Cell Sci 127: 1585-1594, 2014. 
18. Zhang A, Zhou N, Huang J, Liu Q, Fukuda K, Ma D, Lu Z, Bai C, Watabe K and Mo YY: The human long non-coding RNA-RoR is a p53 repressor in response to DNA damage. Cell Res 23: 340-350, 2013

19. Li J, Yu H, Xi M and Lu X: Long noncoding RNA C17orf91 is a potential prognostic marker and functions as an oncogene in ovarian cancer. J Ovarian Res 9: 49, 2016.

20. Marín-Béjar O, Marchese FP, Athie A, Sánchez Y, González J, Segura V, Huang L, Moreno I, Navarro A, Monzó M, et al: Pint lincRNA connects the p53 pathway with epigenetic silencing by the Polycomb repressive complex 2. Genome Biol 14: R104, 2013

21. Ponting CP and Belgard TG: Transcribed dark matter: Meaning or myth? Hum Mol Genet 19: R162-R168, 2010.

22. Gutschner T and Diederichs S: The hallmarks of cancer: A long non-coding RNA point of view. RNA Biol 9: 703-719, 2012.

23. Nie L, Wu HJ, Hsu JM, Chang SS, Labaff AM, Li CW, Wang Y, Hsu JL and Hung MC: Long non-coding RNAs: Versatile master regulators of gene expression and crucial players in cancer. Am J Transl Res 4: 127-150, 2012.

24. Geng YJ, Xie SL, Li Q, Ma J and Wang GY: Large intervening non-coding RNA HOTAIR is associated with hepatocellular carcinoma progression. J Int Med Res 39: 2119-2128, 2011.

25. Ding C, Cheng S, Yang Z, Lv Z, Xiao H, Du C, Peng C, Xie H, Zhou L, Wu J and Zheng S: Long non-coding RNA HOTAIR promotes cell migration and invasion via down-regulation of RNA binding motif protein 38 in hepatocellular carcinoma cells. Int J Mol Sci 15: 4060-4076, 2014.

26. Xu C, Yang $M$, Tian J, Wang $X$ and Li Z: MALAT-1: A long non-coding RNA and its important 3 ' end functional motif in colorectal cancer metastasis. Int J Oncol 39: 169-175, 2011.
27. Yeung TL, Leung CS, Yip KP, Au Yeung CL, Wong ST and Mok SC: Cellular and molecular processes in ovarian cancer metastasis. A review in the theme: Cell and molecular processes in cancer metastasis. Am J Physiol Cell Physiol 309: C444-C456, 2015.

28. Zhao H, Guo L, Zhao H, Zhao J, Weng H and Zhao B: CXCR4 over-expression and survival in cancer: A system review and meta-analysis. Oncotarget 6: 5022-5040, 2015.

29. Furusato B, Mohamed A, Uhlén M and Rhim JS: CXCR4 and cancer. Pathol Int 60: 497-505, 2010.

30. Izumi D, Ishimoto T, Miyake K, Sugihara H, Eto K, Sawayama H, Yasuda T, Kiyozumi Y, Kaida T, Kurashige J, et al: CXCL12/CXCR4 activation by cancer-associated fibroblasts promotes integrin $\beta 1$ clustering and invasiveness in gastric cancer. Int J Cancer 138: 1207-1219, 2016.

31. Guo Q, Gao BL, Zhang XJ, Liu GC, Xu F, Fan QY, Zhang SJ, Yang B and Wu XH: CXCL12-CXCR4 axis promotes proliferation, migration, invasion, and metastasis of ovarian cancer. Oncol Res 22: 247-258, 2014

32. Meng Y, Hu J, Chen Y, Yu T and Hu L: Silencing MARCH1 suppresses proliferation, migration and invasion of ovarian cancer SKOV3 cells via downregulation of NF- $\kappa \mathrm{B}$ and $\mathrm{Wnt} / \beta$-catenin pathways. Oncol Rep 36: 2463-2470, 2016.

This work is licensed under a Creative Commons Attribution-NonCommercial-NoDerivatives 4.0 International (CC BY-NC-ND 4.0) License. 